\title{
Glosario de términos sobre la regulación de la profesión médica
}

\author{
Arcadi Gual, Maria Nolla, Jordi Palés-Argullós, Luis Pallarés
}

\section{Acreditación / Accreditation}

Proceso mediante el cual un organismo comprueba el cumplimiento de los requisitos o estándares de calidad previamente definidos de una actividad de FMC/DPC o de una institución proveedora de actividades de FMC/DPC. Es frecuente que este término se use como sinónimo de 'certificación' de una determinada actividad, aunque el término 'acreditación' no debería utilizarse para personas. Desgraciadamente están institucionalizadas diferentes 'acreditaciones' dirigidas a personas, como 'acreditación de tutores clínicos' o 'diplomas de acreditación y acreditación avanzada', previstos en la LOPS.

Actuación profesional / Performance

Ejercicio de la práctica profesional en contexto real. Aunque es frecuente que el término 'performance' se traduzca por 'desempeño', los términos 'practica profesional', 'ejercicio profesional' o 'actuación profesional' son más adecuados.

Agencia de acreditación / Accreditation body

Entidad responsable de la acreditación de actividades de FMC/DPC en un determinado país o ámbito. Suele ser responsable del reconocimiento de los créditos de formación obtenidos por un profesional de dicho país o ámbito.

En la inmensa mayoría de países o ámbitos con sistemas de acreditación, estas organizaciones son específicas para cada profesión.

\section{Agencia reguladora / Regulatory body}

Entidad responsable de la regulación de la profesión médica en un determinado ámbito o país.

Su naturaleza puede ser, básicamente, gubernamental, profesional o mixta.

\section{Análisis de necesidades / Needs assessment}

Proceso que estudia la diferencia entre las competencias reales y las deseables de un profesional según cada contexto y momento histórico.

\section{Auditoría clínica / Clinical audit}

Revisión sistemática de registros clínicos que reflejan la labor asistencial comparándola con determinados estándares previamente definidos y aceptados como criterios de referencia.

Su propósito puede ser identificar áreas de mejora o certificar unos mínimos competenciales.
Aprendizaje autónomo / Self-learning

También llamado autoaprendizaje, es el proceso por el cual la persona adquiere o desarrolla competencias asumiendo su responsabilidad y escogiendo las estrategias educativas que le son más útiles.

\section{Bienes de confianza / Credence goods}

Bienes donde es muy difícil que el consumidor compruebe el nivel de calidad que le han proporcionado tanto ex ante o durante la prestación del servicio, como ex post.

Responden a las características de los servicios profesionales cuyo ejemplo paradigmático son los servicios sanitarios.

Búsqueda de rentas / Rent-seeking

En la teoría de la elección pública, es la situación que se produce cuando un individuo, organización o empresa busca obtener ingresos captando renta económica a través de la manipulación o explotación del entorno político o económico, en lugar de obtener beneficios a través de transacciones económicas y producción de riqueza añadida.

La mayoría de los estudios de búsqueda de rentas se centran en los esfuerzos para captar privilegios especiales en monopolios, como la regulación gubernamental de la libre competencia de empresas.

\section{Captura / Capture}

Actividad de presión ejercida por grupos profesionales con la intención de influenciar al gobierno para que dicte leyes que les beneficien.

\section{Carrera profesional / Professional career}

Internacionalmente se entiende como un reconocimiento no jerárquico y reversible de unas funciones o responsabilidades específicas dentro de una institución u organización asistencial en el marco de su política de recursos humanos. En España viene definida en la Ley del Estatuto Marco del personal estatutario de los servicios de salud, de 16 de diciembre de 2003, como el derecho de los profesionales a progresar, de forma individualizada, como reconocimiento a su desarrollo profesional en cuanto a conocimientos, experiencia y cumplimiento de los objetivos de la organización en la que prestan sus servicios.

La formulación operativa de la carrera profesional depende de los gobiernos de las comunidades autónomas.
Coordinación/Correspondencia: Fundación Educación Médica. Departamento de Ciencias Fisiológicas I. Facultad de Medicina. Universitat de Barcelona. Casanova, 143. E-08036 Barcelona.

E-mail:

agual@

fundacioneducacionmedica.cat

(c) 2013 FEM 


\section{Categorías de créditos de FMC/DPC / \\ CME/CPD credit categories}

Agrupación de los créditos otorgados a las actividades de FMC/DPC en función del tipo de actividad, la población diana o el nivel de calidad educativa.

En España, el Sistema Nacional de Formación Continuada sólo ha desarrollado un tipo de crédito (basado en su duración, 10 horas un crédito, y ponderado por un factor de calidad).

\section{Certificación profesional / Professional certification}

Es una credencial conseguida por un médico y emitida por un ente certificador que garantiza, tras su comprobación, su cualificación para realizar un trabajo o tarea; frecuentemente se la denomina 'certificación.'

Actualmente se acepta la necesidad de renovar periódicamente las certificaciones, utilizándose el término 'recertificación’ para la renovación periódica de una certificación. El término 'certificación' no debería utilizarse para instituciones o actividades; en estos casos debería emplearse el término 'acreditación'

Colegiación / Registration (Reino Unido y otros) /

Licensing (EE. UU.)

Inscripción de un médico en el registro colegial de naturaleza obligatoria para ejercer la asistencia en España. Es requisito para los licenciados/graduados del Estado Español el título de licenciado/graduado en Medicina; para un licenciado/graduado de un país comunitario es requisito el título de licenciado/graduado en Medicina previa 'credencial de reconocimiento de valor comunitario'; y para un licenciado/graduado de un país no comunitario son requisitos la credencial de homologación del título de licenciado/graduado en Medicina y el título de licenciado/graduado en Medicina.

\section{Colegios de médicos / College of Physicians} Corporaciones de derecho público, reguladas por la Ley de Colegios Profesionales y las disposiciones legislativas aprobadas por los gobiernos autonómicos, cuyas funciones se centran en la ordenación del ejercicio de los médicos, la representación y defensa de sus intereses profesionales, la salvaguarda y observancia de los principios deontológicos y ético-sociales por parte de los mismos, la promoción de la constante mejora de sus niveles científico, cultural y económico, y la colaboración con los poderes públicos en la consecución del derecho a la protección de la salud de todos los españoles y la más eficiente, justa y equitativa regulación de la asistencia sanitaria y del ejercicio de la medicina.

En la actualidad, la colegiación es obligatoria en la mayoría de las comunidades autónomas, y en las que era voluntaria para los profesionales con ejercicio exclusivo en la sanidad pública, diferentes sentencias han confirmado la obligatoriedad de colegiación para el ejercicio profesional de los médicos. El actual Anteproyecto de Ley de Servicios y Colegios Profesionales contempla la obligatoriedad de la colegiación para los médicos.

\section{Colusión / Collusion}

En economía se denomina al acuerdo en que dos o más empresas de un mercado determinado definen que cada una actuará de manera concertada respecto del resto de las demás empresas.

El fin de este tipo de acuerdos es que cada una de las empresas participantes en la colusión tome control de una determinada porción del mercado en el que operan, y ac- tuar de manera monopolista, impidiendo a otras empresas entrar en el mercado.

\section{Competencia / Competence}

Constructo de orden superior fruto de la adquisición y desarrollo de conocimientos, habilidades, actitudes y valores necesarios para llevar a cabo una función en un contexto y momento determinados.

Conflicto de interés / Conflict of competing interest Situación que se le plantea a un médico frente a una decisión en la que el interés del paciente puede no anteponerse al del propio médico, al de la institución sanitaria en la que presta sus servicios o a cualquier organización comercial o industrial.

Corregulación profesional / Professional co-regulation Véase 'regulación profesional compartida'.

\section{Credencial / Credentials}

Orden o documento que atestigua o autoriza la cualificación, competencia o autoridad otorgada a un individuo por un tercero, sin perjuicio de obtener luego el título correspondiente.

Ejemplos de credenciales son: los diplomas, los títulos académicos, los títulos de grado, los documentos de identidad o de conducir, etc. En algunos países hispanohablantes sólo se denomina credencial a algunos de estos documentos y no a otros.

\section{Credencialismo / Credentialism}

Término usado para describir la 'confianza en' o la 'prioridad de' las credenciales.

A menudo las credenciales se han utilizado en exceso a expensas de la cualificación o competencia para la asignación de tareas o condición social, por lo que, en ocasiones, dicho término ha devenido despectivo refiriéndose al mal uso de las credenciales.

\section{Crédito de FMC/DPC / CME/CPD credit}

Unidad de valoración de una actividad de FMC/DPC determinada por diferentes mecanismos, en los que como mínimo se incluyen el número de horas lectivas y la apreciación de calidad.

\section{Crédito europeo de FMC/DPC /}

\section{CME/CPD European credits}

Créditos de FMC/DPC otorgados por el European Accreditation Council for Continuing Medical Education $\left(\right.$ EACCME $\left.^{\circledR}\right)$, el organismo acreditador de la UEMS.

Esta iniciativa de la UEMS permite el reconocimiento y el intercambio de créditos de FMC/DPC entre todos los países europeos; además, UEMS-EACCME tiene acuerdos de reconocimiento mutuo con la Asociación Médica Americana tanto para actividades presenciales como para actividades a distancia, y con el Real Colegio de Médicos y Cirujanos de Canadá sólo para actividades presenciales.

Desarrollo profesional continuo (DPC) /

\section{Continuing professional development (CPD)}

Conjunto de todas las actividades orientadas a la actualización, desarrollo y mejora de la competencia requerida para una adecuada práctica clínica a lo largo de la vida profesional, y comprende fundamentalmente tres tipos de actividades: el aprendizaje autónomo, el aprendizaje en servicio y las actividades de formación médica continuada.

En España, la LOPS define el desarrollo profesional continuo como el reconocimiento público, expreso y de forma individualizada, del desarrollo alcanzado por un profesional sani- 
tario en cuanto a conocimientos, experiencia en las tareas asistenciales, docentes y de investigación, así como en cuanto al cumplimiento de los objetivos asistenciales e investigadores de la organización en la que prestan sus servicios.

Diplomas de acreditación y diplomas

de acreditación avanzada / N/A

Contemplados en la LOPS como certificaciones del nivel de formación alcanzado por un profesional sanitario en una área funcional específica de una determinada profesión o especialidad, en función de las actividades de formación continuada acreditada desarrolladas por el interesado en el área funcional correspondiente.

\section{ECMEC / ECMECs}

Véase 'crédito europeo de FMC/DPC'.

\section{Ente acreditador / Acreditating body}

Organismo encargado de implementar procesos de acreditación, programas o centros, y que tiene garantizada su independencia para emitir un juicio.

Frecuentemente, estos entes $u$ organismos se concretan en agencias, consejos o fundaciones y pueden ser tanto públicos como privados, aunque en el primer caso deben extremarse los controles para garantizar su independencia.

Ente certificador / Certificating body

Organismo encargado de implementar procesos de certificación de profesionales y que tiene garantizada su independencia para emitir un juicio.

Frecuentemente, estos entes $u$ organismos se concretan en agencias, consejos o fundaciones y pueden ser tanto públicos como privados, aunque en el primer caso deben extremarse los controles para garantizar su independencia.

Evaluación / Assessment

Proceso de recogida de información, emisión de un juicio de valor, según unos criterios previamente definidos, para tomar una decisión.

El termino original en inglés para esta definición, 'assessment', tiene varios sinónimos, por lo que en castellano hay dificultades a la hora de distinguir entre 'appraisal', 'assessment' y 'evaluation'.

Evaluación de la actuación profesional /

\section{Performance evaluation}

Medida de la práctica de un profesional en un momento concreto, comparándola con estándares predeterminados, que permite establecer medidas de mejora de la competencia.

\section{Experteza / Expertise}

Anglicismo que explica una actuación de alta calidad fruto de la experiencia.

La traducción literal del término 'expertise' correspondería a 'pericia'.

Formación médica continuada (FMC) /

Continuing medical education (CME)

Conjunto de actividades educativas orientadas a la adquisición, mantenimiento o mejora de la competencia profesional.

En España, la LOPS define la formación continuada como el proceso de enseñanza y aprendizaje activo y permanente al que tienen derecho y obligación los profesionales sanitarios, que se inicia al finalizar los estudios de pregrado o de especialización y que está destinado a actualizar y mejorar los conocimientos, habilidades y actitudes de los profesionales sanitarios ante la evolución científica y tecnológica y las demandas y necesidades, tanto sociales como del propio sistema sanita- rio. De manera genérica, la formación médica continuada se suele definir como el conjunto de actividades educativas que lleva cabo el médico una vez finalizado su período de pregrado y de especialización, para mantener actualizada su competencia, y que no comportan una titulación adicional.

Institución con ánimo de lucro / Commercial organization Entidad que proporciona servicios de diferentes tipos (sanitarios, educativos, etc.) distribuyendo los excedentes entre los propietarios.

Institución sin ánimo de lucro / Non profit organization Entidad que proporciona servicios de diferentes tipos (sanitarios, educativos, etc.) en la que los excedentes se reinvierten en la propia organización.

Licencia / License Autorización administrativa para el ejercicio de una determinada actividad profesional.

Actualmente, según la normativa española puede equipararse a 'colegiación' o a 'registro'

Organización profesional médica / Medical association Corporación de médicos responsable de actividades profesionales en su propio campo de experteza o pericia.

En España destacan los colegios de médicos y las sociedades científicas.

Par / Peer

Persona que ocupa la misma posición o cargo que otra dentro de una organización.

Se puede traducir por 'igual' o 'colega'.

\section{Portafolio / Portfolio}

Documento que recoge de forma más o menos sistemática y estructurada elementos que evidencian el aprendizaje o nivel de competencia conseguido según unos objetivos establecidos previamente, y siempre acompañado de un proceso de reflexión.

Principios del profesionalismo médico /

Principles of medical professionalism

Los principios clásicos del profesionalismo médico son cuatro: beneficencia, no maleficencia, autonomía y justicia. En 2002, las sociedades profesionales anglosajonas consensuaron tres principios: el de primacía de bienestar del paciente, el de autonomía del paciente y el de justicia social.

En cualquier caso, el elemento común de los principios para el ejercicio de la profesión médica radica en anteponer los intereses del paciente a los del propio médico como base de la confianza que el paciente deposita en el médico y en la profesión médica.

\section{Profesión / Profession}

Ocupación que tiene un cuerpo de conocimientos altamente especializados, capacidad de autorregulación, espíritu de servicio a la sociedad, código deontológico y reconocimiento social.

\section{Profesión médica / Medical profession}

Ocupación basada en el desempeño de tareas encaminadas a afrontar problemas de salud y a identificar y diagnosticar enfermedades aplicando un cuerpo de conocimiento especializado propio de nivel superior, en la que preside el espíritu de servicio y en la que se persigue el beneficio del paciente antes que el propio, y para la cual se requiere que las partes garanticen: 1) la producción, el uso y la transmisión del conocimiento científico; 2) la mejora permanente para prestar la mejor asistencia posible; 3 ) la aplicación del conocimiento de 
forma ética y competente, y 4) que la práctica profesional se oriente hacia las necesidades de salud y de bienestar de las personas y de la comunidad.

\section{Profesional médico / Medical professional}

Médico, o médica, titulado/a comprometido/a con los principios éticos y deontológicos y los valores de la profesión médica y cuya conducta se ciñe a dichos principios y valores.

\section{Profesionalismo / Professionalism}

Anglicismo con el que se denomina al conjunto de valores, conductas y relaciones que sustentan la confianza de la sociedad en una profesión.

La palabra 'profesionalidad', definida por la Real Academia Española como 'cualidad de la persona u organismo que ejerce su actividad con relevante capacidad y aplicación', no incluye matices fundamentales de la palabra inglesa 'professionalism?

Profesionalismo médico / Medical professionalism Conjunto de principios éticos y deontológicos, valores y conductas que sustentan el compromiso de los profesionales de la medicina con el servicio a los ciudadanos, que evolucionan con los cambios sociales, y que avalan la confianza que la población tiene en los médicos y sustentan el contrato tácito entre la sociedad y los profesionales de la medicina.

\section{Recertificación / Re-certification}

Proceso periódico para renovar una credencial conseguida y que garantiza su cualificación para el ejercicio profesional.

Habitualmente es atribuida a la renovación de las competencias específicas de una especialidad médica.

Recolegiación / Re-registration (Reino Unido y otros) /

\section{Re-licensing (EE. UU.)}

Proceso periódico por el que un colegio profesional renueva la credencial de la colegiación.

En España no existe este proceso y se ha estructurado uno equivalente, de naturaleza voluntaria, denominado 'validación periódica de la colegiación' (VPC).

\section{Regulación profesional / Professional regulation}

Proceso propio de las profesiones que consiste en establecer unos criterios o estándares tanto para el acceso a la corporación profesional como para permanecer en la misma, y cuyo fin es proporcionar garantías y protección a la ciudadanía respecto a la calidad de los actos de una determinada profesión o colectivo.

Así pues, se dice que una profesión está regulada cuando el acceso y el ejercicio están sujetos a la posesión y comprobación de una cualificación profesional específica.

Regulación profesional compartida /

\section{Shared professional regulation}

Proceso de regulación profesional en el que intervienen dos o más agentes reguladores.

\section{Revalidación / Revalidation}

Proceso de volver a validar.

Es frecuente que diferentes tipos de validaciones tengan una duración temporal predeterminada, por lo que requieren ser validadas periódicamente.

Revisión por pares / Peer review

Revisión de una actividad educativa, de un material cien- tífico o de una actuación profesional por parte de otros que son expertos en el mismo campo.

En España suele utilizarse tanto el término 'revisión por pares' como 'revisión por colegas'.

Riesgo moral / Moral hazard

Concepto económico que describe las situaciones en las que un individuo conoce las consecuencias de sus acciones y, sin embargo, son otras personas las que soportan las consecuencias de los riesgos asumidos. El riesgo moral informa cómo los individuos asumen mayores riesgos cuando las posibles consecuencias negativas de sus actos no son asumidas por ellos, sino por un tercero. Existe riesgo moral cuando una persona tiene una mayor información acerca de sus propias acciones que el resto de los individuos (información asimétrica). El riesgo moral reduce la capacidad del mercado para asignar eficientemente el riesgo.

Sesgo comercial / Comercial bias

Desviación de los contenidos de una actividad educativa, investigadora o asistencial en favor de los intereses del patrocinador comercial de ésta.

Sociedades científicas / Scientific societies

Entidades de derecho privado que agrupan a los médicos en función de su ámbito de especialización, cuyos fines son la promoción del conocimiento científico, la investigación en sus áreas de competencia y la formación continuada y el desarrollo profesional de sus miembros.

La afiliación es de carácter voluntario.

\section{Validación / Validation}

Proceso mediante el cual un ente comprueba que se cumplen unos requisitos o estándares previamente establecidos y garantiza un nivel de calidad

Un proceso de validación puede referirse a personas, instituciones o actividades, y normalmente origina una credencial ad hoc. Ejemplos de procesos de validación serían las certificaciones o las acreditaciones.

\section{Validación periódica de la colegiación / $N / A$}

Proceso voluntario y periódico (cada seis años) por el que los colegios de médicos españoles renuevan a sus colegiados la credencial de la colegiación tras evaluar la buena praxis médica, el buen estado psicofísico para el ejercicio de su actividad profesional y el buen desempeño profesional.

Sería un ejemplo concreto de recertificación.

\section{Valoración / Appraisal}

Proceso de evaluación de la competencia de un profesional, generalmente realizada por el profesional y su superior jerárquico, que incluye la reflexión sobre la actuación individual, la identificación de sus necesidades de formación y el diseño de planes para su desarrollo personal. Se focaliza pues en el propio profesional y sus necesidades educativas.

En castellano, el término inglés 'appraisal' es sinónimo de evaluación, estimación, apreciación o valoración, pero se utiliza para los casos en los que dicha evaluación se centra en las necesidades individuales.

$\mathrm{VPC} / \mathrm{N} / \mathrm{A}$

Véase 'validación periódica de la colegiación'. 